\title{
Clocking Ultrafast Wave Packet Dynamics in Molecules through UV-Induced Symmetry Breaking
}

\author{
Alberto González-Castrillo, ${ }^{1}$ Alicia Palacios, ${ }^{1}$ Henri Bachau, ${ }^{2}$ and Fernando Martín ${ }^{1,3}$ \\ ${ }^{1}$ Departamento de Química, Módulo 13, Universidad Autónoma de Madrid, 28049 Madrid, Spain \\ ${ }^{2}$ Centre des Lasers Intenses et Applications, CNRS-CEA-Université de Bordeaux 1, F-33405, Talence, France \\ ${ }^{3}$ Instituto Madrileño de Estudios Avanzados en Nanociencia (IMDEA-Nanociencia), Cantoblanco, 28049 Madrid, Spain
}

(Received 28 September 2011; published 10 February 2012)

\begin{abstract}
We investigate the use of UV-pump-UV-probe schemes to trace the evolution of nuclear wave packets in excited molecular states by analyzing the asymmetry of the electron angular distributions resulting from dissociative ionization. The asymmetry results from the coherent superposition of gerade and ungerade states of the remaining molecular ion in the region where the nuclear wave packet launched by the pump pulse in the neutral molecule is located. Hence, the variation of this asymmetry with the time delay between the pump and the probe pulses parallels that of the moving wave packet and, consequently, can be used to clock its field-free evolution. The performance of this method is illustrated for the $\mathrm{H}_{2}$ molecule.
\end{abstract}

PACS numbers: 33.80.- b, 82.53.Eb

The rapid development of ultrashort laser pulses combined with optical pump-probe spectroscopy has opened the way for controlling and manipulating electron (and nuclear) dynamics in atoms (and molecules). For molecules, recent advances in coherent pulse control, as carrierenvelope phase stabilization, has triggered the interest in steering and tracing electron motion upon ionization by means of intense IR fields [1-7]. They have also suggested that the analysis of the vibrational population of the remaining molecular ion can be used as a clock for the recollision events [5,6]. More recent experiments have made use of few-cycle IR probe pulses on molecules ionized by a single attosecond pulse [8] or a train of attosecond pulses $[9,10]$. In all the above experiments, $\mathrm{H}_{2}$ and $\mathrm{D}_{2}$ molecular targets were used, which is the natural choice in view of the many variables potentially intervening in these kinds of problems and the need for theoretical support to understand the ensuing dynamics and to suggest alternative mechanisms of control $[8,10,11]$.

In the control schemes mentioned above, the dynamics is mostly driven by the strong IR field. An alternative procedure that allows one to explore field-free molecular dynamics is to use XUV pump-XUV probe schemes $[12,13]$, which have been successfully employed to image nuclear wave packets (NWPs) of the $\mathrm{D}_{2}^{+}$ion in experiments leading to the Coulomb explosion (CE) of $\mathrm{D}_{2}$ $[14,15]$. Free electron lasers provide such femtosecond (fs) pulses in vacuum ultraviolet and soft x-ray energy domains [16] and new facilities are expected to generate fs pulses in a wider photon energy range $(12-124 \mathrm{eV})$ [17]. In spite of the relatively high intensity of the light generated by these lasers, its short wavelength ensures that the field-induced potential is much smaller than the electron-nucleus and electron-electron potentials (perturbative regime [18]), so the dynamics of the NWP is exclusively driven by the unperturbed molecular potential.
In this work we focus on the use of UV pulses to trace the NWP generated in excited states of neutral molecules. We will show how to use a UV-pump-UV-probe scheme with identical single pulses to trace the evolution of the NWP generated by the pump pulse in the excited states of $\mathrm{H}_{2}$. The method relies on the analysis of the symmetry breaking of the electron angular distributions resulting from dissociative ionization of $\mathrm{H}_{2}$ by the combination of both pulses. The up-down (or left-right) asymmetry of electron localization in molecules that dissociatively ionize is a well-known process [19-21] that arises from the superposition of two states with different parity. Existing experiments have used this strategy to map electron localization induced by the IR field in both the double ionization channel [3-6] (i.e., CE) and the single ionization channel [2,7-9]. Symmetry breaking of electron angular distributions has also been observed in single photoionization of $\mathrm{H}_{2}$, but, in this case, the asymmetry provides information about the delayed electron emission from the molecular doubly excited states [22]. In this work we show that studying the variation of the electron angular distribution asymmetries with the time delay between the pump and probe UV pulses can also provide a clear image of the time evolution of the wave packet generated by the pump pulse in excited states of the neutral molecule. This is relevant because such evolution is often obscured in the kinetic energy release (KER) spectra by contributions from several ionization channels, or by the presence of complex structures resulting from interferences between energydegenerate paths, or by the broad bandwidth of the ultrashort pulses.

We will only consider linearly polarized light parallel to the molecular axis. The theoretical method has been explained in detail in previous works [23,24]. Briefly we solve the time-dependent Schrödinger equation in full dimensionality by expanding the time-dependent wave 
function in a basis of fully correlated $\mathrm{H}_{2}$ vibronic stationary states of $\Sigma_{g}^{+}$and $\Sigma_{u}^{+}$symmetries built upon the bound electronic states, the nonresonant electronic continuum states associated with the $1 s \sigma_{g}$ and $2 p \sigma_{u}$ ionization channels, and the lowest $Q_{1}$ and $Q_{2}$ series of doubly excited states. The electronic part of the vibronic wave function is calculated in a box of 60 a.u. and the nuclear part in a box of 12 a.u. The size of these boxes is large enough to ensure that there are no significant reflections of electronic and nuclear wave packets in the box boundaries for propagation times smaller than $\tau+T$, where $T$ is the duration of the UV pulses and $\tau$ is the time delay (from maximum to maximum). Pump and probe pulses are identical with a central frequency of $12.2 \mathrm{eV}$, duration $T=2 \mathrm{fs}$ and cosine square temporal envelope. The intensity is $10^{12} \mathrm{~W} \mathrm{~cm}^{-2}$. Apart from truncation of the basis of vibronic states at a high enough energy $(\sim 65 \mathrm{eV}$ above the $\mathrm{H}_{2}$ ground state), the only approximations made in our calculations are (i) the dipole approximation in describing the laser-molecule interaction and (ii) the neglect of nonadiabatic couplings and molecular rotations. In previous work, this method has been shown to yield integral and differential cross sections for dissociative ionization of $\mathrm{H}_{2}$ and $\mathrm{D}_{2}$ in good agreement with various experimental measurements (see, e.g., $[8,10,25]$ ).

The chosen field intensity is low enough so that distortion of the molecular potential by the external field is negligible (e.g., such a field cannot lead to bond softening, which usually appears at low proton kinetic energy releases, $\sim 0.5 \mathrm{eV}$, in intense infrared fields [26]). In our scheme, the probability of double ionization is orders of magnitude smaller than that of single ionization (the double ionization potential of $\mathrm{H}_{2}$ in a vertical transition from the ground state is $\sim 51 \mathrm{eV}$ and, therefore, would require the unlikely absorption of four photons). The energetics and relevant states involved in this process are plotted in Fig. 1. The pump pulse generates a vibrational wave packet in the first excited state of the neutral $\left(B^{1} \Sigma_{u}^{+}\right)$. The wave packet starts to spread and is probed at different times.

The variation of the electron angular distributions with time delay is shown in Fig. 2 for three values of the proton kinetic energy release (KER): $0.5,1.1$, and $1.5 \mathrm{eV}$. The angular distributions are integrated over all electron energies and are normalized to that corresponding to a delay of $4 \mathrm{fs}$ and a KER of $0.5 \mathrm{eV}$ (dotted line in the left panel). The figure clearly shows that, for each value of the KER, the angular distributions become clearly asymmetric at a given time delay: around 10,8 , and 6 fs for a KER of $0.5,1.1$, and 1.5 , respectively. KER spectra for dissociative single ionization are plotted in Fig. 3. The figure also shows the partial contributions of the ionization channels associated with the ground $\left(1 s \sigma_{g}\right)$ and first excited $\left(2 p \sigma_{u}\right)$ states of the $\mathrm{H}_{2}^{+}$ion. Vertical lines in Fig. 3 indicate the values of the KER selected in Fig. 2. As can be seen, at zero time delay

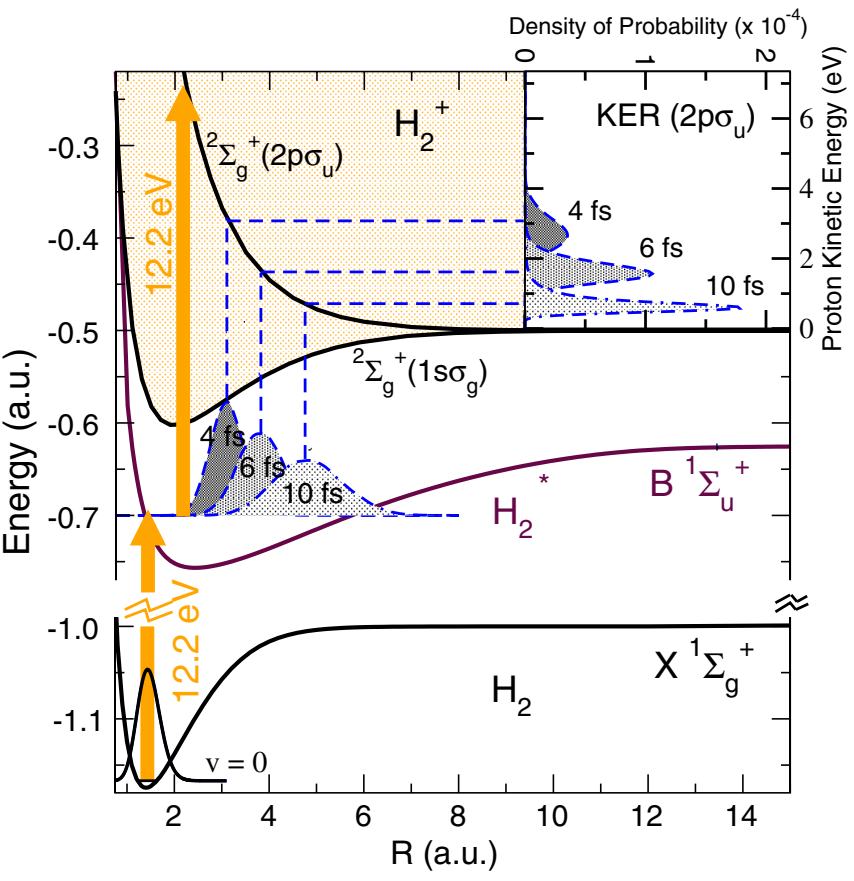

FIG. 1 (color online). Relevant potential energy curves for $\mathrm{H}_{2}$ and the $\mathrm{H}_{2}^{+}$ion. Pump and probe pulses (with central frequency of $12.2 \mathrm{eV}$ ) are indicated with arrows. Calculated squared amplitudes of the nuclear wave packet generated by the pump pulse after 4, 6, and $10 \mathrm{fs}$ are shown as functions of the internuclear distance. Dashed lines indicate (i) the vertical transition from their maximum to the second ionization threshold $\left(2 p \sigma_{u}\right)$ and (ii) the expected energies in the continuum (horizontal dashed lines) connected to the actual kinetic energy releases shown in the inset for that threshold.

(top panel in Fig. 3), ionization through the ground state of the ion is the only contribution to dissociative ionization. As time delay increases, one observes that contribution of the $2 p \sigma_{u}$ ionization channel becomes progressively more apparent and moves to lower KERs.

The observed behavior is the direct consequence of the evolution of the vibrational wave packet launched by the pump pulse in the $B^{1} \Sigma_{u}^{+}$state. Figure 1 shows this wave packet 4,6 , and $10 \mathrm{fs}$ after its creation. As can be seen, there is an almost perfect one-to-one correspondence between the position of the vibrational wave packet at a given time and the actual KER spectrum associated with dissociative ionization in the $2 p \sigma_{u}$ channel (see the inset in Fig. 1). Thus, the time delay between the pump and the probe is a measure of the traveling time of the NWP in the $B^{1} \Sigma_{u}^{+}$state. This is not surprising since a similar correspondence between NWPs and KER spectra has been observed in the $\mathrm{CE}$ of $\mathrm{H}_{2}$ following double ionization [15]. In the latter case, the NWP can be reconstructed almost perfectly by using the so called reflection approximation [27] in which the measured KER distribution is transformed into the actual wave packet by simply convoluting this distribution with the $1 / R$ repulsive potential 


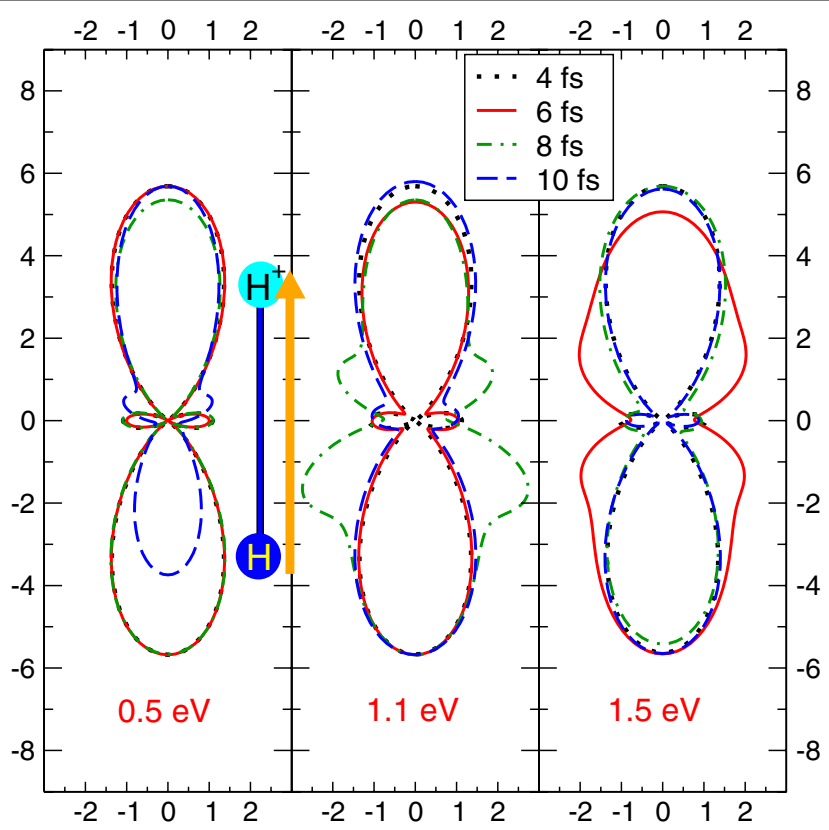

FIG. 2 (color online). Electron angular distributions integrated over all electron energies at different time delays between pulses. Each panel corresponds to a given proton kinetic energy release $(0.5,1.1$ and $1.5 \mathrm{eV}$, respectively). Probability distributions are normalized to that obtained for a time delay of $4 \mathrm{fs}$ and a KER of $0.5 \mathrm{eV}$ (dotted line in the left panel). Values are given in units of $10^{-5}$.

energy curve. Thus, inspection of the KER spectrum resulting from CE provides a direct picture of the NWP motion. In the present case, reconstruction of the NWP is also possible by using the KER distribution associated with the $2 p \sigma_{u}$ channel and replacing the $1 / R$ curve by the $2 p \sigma_{u}$ one. However, this has little practical interest here because, in contrast with $\mathrm{CE}$ in which a single channel is involved, dissociative ionization resulting from the present pumpprobe scheme involves not only the $2 p \sigma_{u}$ channel but also the $1 s \sigma_{g}$ one. Contributions from both channels overlap in the whole KER spectrum and for all time delays. Therefore, unless the $2 p \sigma_{u}$ contribution dominates the KER spectrum (so that one can easily follow its variation with time delay), extraction of the NWP dynamics from this spectrum may be a difficult task. This problem is even more dramatic when the wave packet created in the $B^{1} \Sigma_{u}^{+}$ state approaches the outer classical turning point, i.e., for a time delay larger than $10 \mathrm{fs}$, because structures due to interferences with direct ionization channels are prominent in the $1 s \sigma_{g}$ channel and measurements at low KER are usually difficult.

An important aspect of the pump-probe scheme considered in this work is that dissociative ionization involves $\mathrm{H}_{2}^{+}$states of different parity, $1 s \sigma_{g}$ and $2 p \sigma_{u}$. A coherent superposition of these states can be observed in measurements that distinguish between protons emitted upwards and protons emitted downwards, which is experimentally

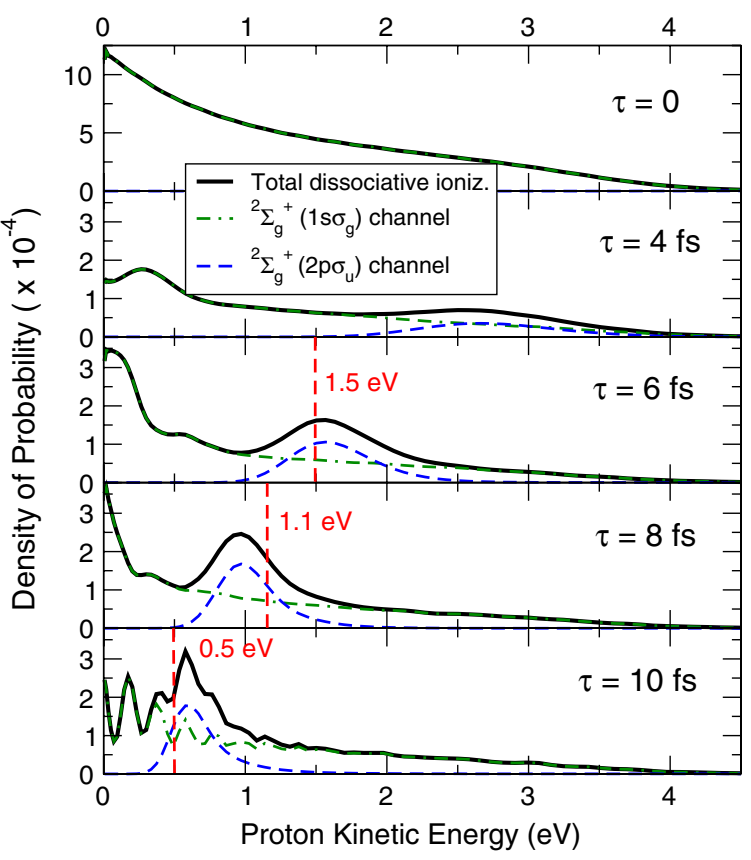

FIG. 3 (color online). Proton kinetic energy distributions for different time delays (labeled in each panel). Solid black lines: total dissociative ionization. Dash-dotted lines: $1 s \sigma_{g}$ contribution. Dashed lines: $2 p \sigma_{u}$ contribution. Vertical dashed lines indicate the kinetic energy releases for the angular distributions plotted in Fig. 2.

achievable by using, e.g., COLTRIMS techniques [28]. Under these circumstances, one can expect that, for a chosen proton- $\mathrm{H}$ geometry, e.g., proton up $\mathrm{H}$ down as in Fig. 2, molecular-frame electron angular distributions become asymmetric when the two channels overlap significantly in the KER spectrum [22], i.e., in the region where the $2 p \sigma_{u}$ contribution exhibits its maximum. Elsewhere, the electron angular distributions should remain nearly symmetric. Indeed, Fig. 3 shows that the largest overlap at a KER of $\sim 1.5,1.1$ and $0.5 \mathrm{eV}$ occurs for delays of 6,8 , and $10 \mathrm{fs}$, respectively, in agreement with the results shown in Fig. 2. Thus, observation of a pronounced asymmetry in the electron angular distribution at a given time delay is a direct measure of the NWP traveling time in the $B^{1} \Sigma_{u}^{+}$ state. It is important to emphasize that if the measurements did not distinguish between up and down protons, the electron angular distributions would be always symmetric due to the lack of coherence between the $g$ and $u$ states.

Measuring the whole electron angular distribution at a given KER is usually a complicated task. Instead it is much more convenient to measure the electron asymmetry parameter, defined as $A=\left(N_{\text {up }}-N_{\text {down }}\right) /\left(N_{\text {up }}+N_{\text {down }}\right)$, where $N_{\text {up }}\left(N_{\text {down }}\right)$ is the probability of ejecting an electron upwards (downwards) [3,8]. These probabilities are obtained by separately integrating the electron angular distributions over the solid angle in the two hemispheres defined by a plane that contains the inversion symmetry 


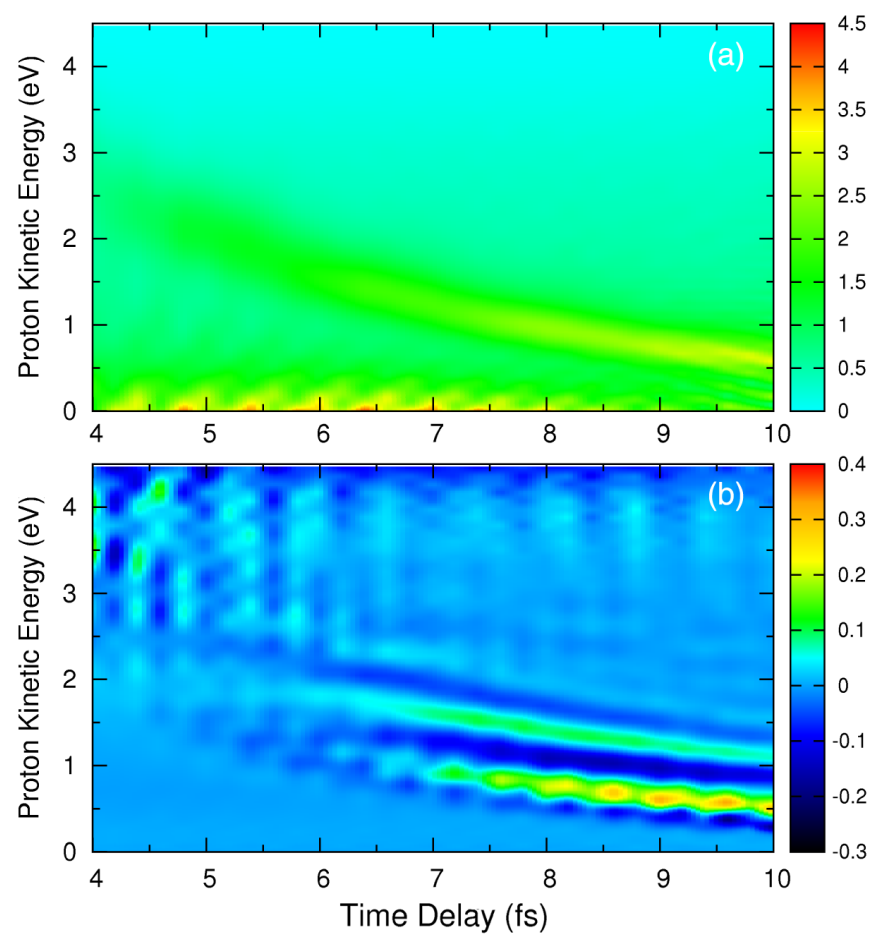

FIG. 4 (color online). (a) Dissociative ionization probability $\left(\times 10^{-4}\right)$ and (b) asymmetry parameter as a function of time delay ( $x$ axis) and proton kinetic energy ( $y$ axis).

center and is perpendicular to the molecular axis. A twodimensional plot of $A$ as a function of KER and time delay is shown in Fig. 4(b). For completeness, Fig. 4(a) shows a similar two-dimensional plot for the dissociative ionization probability (DIP). As can be seen, the evolution of the NWP is quite clear in the asymmetry parameter plot, while it is hardly visible in the DIP plot for the reasons explained above. The high contrast of the image in the asymmetry plot is due to $A$ changing abruptly (from around +0.3 to $-0.3)$ in the KER region where contribution from the $2 p \sigma_{u}$ channel is most important. In contrast, the variations in the DIP plot are due to a factor of 2 at most. In practice, in many state-of-the art experiments, modulations in the two-dimensional DIP plot (or the KER spectrum) as small as those found in this example are not visible (see, e.g., [8]), but those obtained for the molecular-frame asymmetry parameter should be clearly visible (in [8], laboratoryframe asymmetry parameters involving similar variations and roughly half of the events required to determine the present molecular-frame asymmetry parameters have been clearly observed ). Following the evolution of the NWP in the DIP plot is even more difficult for pulses shorter than those used in the present work because, due to their large bandwidth, probability distributions are even wider and therefore exhibit less pronounced maxima. Furthermore, the spreading of the NWP with time delay leads to progressively wider kinetic energy distributions, which makes identification of maxima even more difficult (the NWP takes around $30 \mathrm{fs}$ to make a complete round trip, as shown by recent two-color UV-pump-UV-probe simulations [29]).

In summary, we have shown how to use UV-pump-UVprobe schemes to trace the evolution of nuclear wave packets in excited states of $\mathrm{H}_{2}$ by analyzing the asymmetry of the electron angular distributions resulting from dissociative ionization of the molecule. Since this asymmetry results from the coherent superposition of $\mathrm{H}_{2}^{+}$states of different parity and appears in the region of the KER spectrum where the corresponding ionization channels overlap, it allows one to easily retrieve the position of the pumped nuclear wave packet. Thus, the variation of this asymmetry with the pump-probe time delay can be used to clock the field-free evolution of the wave packet.

This work was accomplished with an allocation of computer time from Mare Nostrum BSC and CCC-UAM, and was partially supported by the MICINN Projects No. FIS2010-15127, No. ACI2008-0777 and No. CSD 2007-00010, the ERA-Chemistry Project No. PIM2010EEC-00751, the European grants MC-ITN CORINF and MC-RG ATTOTREND, the European COST Action CM0702, and the Advanced Grant of the European Research Council XCHEM 290853. A. P. acknowledges a Juan de la Cierva contract grant from MICINN.

[1] C. Calvert, W. Bryan, W. Newell, and I. Williams, Phys. Rep. 491, 1 (2010).

[2] B. Fischer, M. Kremer, T. Pfeifer, B. Feuerstein, V. Sharma, U. Thumm, C. Schröter, R. Moshammer, and J. Ullrich, Phys. Rev. Lett. 105, 223001 (2010).

[3] M. F. Kling et al., Science 312, 246 (2006).

[4] H. Niikura, D. M. Villeneuve, and P. B. Corkum, Phys. Rev. A 73, 021402 (2006).

[5] H. Niikura, F. Legare, R. Hasbani, M. Ivanov, D. Villeneuve, and P. Corkum, Nature (London) 421, 826 (2003).

[6] H. Niikura, F. Légaré, R. Hasbani, A. D. Bandrauk, M. Y. Ivanov, D. M. Villeneuve, and P. B. Corkum, Nature (London) 417, 917 (2002).

[7] M. Kremer, B. Fischer, B. Feuerstein, V. L. B. de Jesus, V. Sharma, C. Hofrichter, A. Rudenko, U. Thumm, C. D. Schröter, and R. Moshammer et al., Phys. Rev. Lett. 103, 213003 (2009).

[8] G. Sansone et al., Nature (London) 465, 763 (2010).

[9] K. P. Singh et al., Phys. Rev. Lett. 104, 023001 (2010).

[10] F. Kelkensberg et al., Phys. Rev. Lett. 107, 043002 (2011).

[11] G. Sansone, F. Kelkensberg, F. Morales, J. F. Pérez-Torres, F. Martín, and M. J. J. Vrakking, IEEE J. Sel. Top. Quantum Electron. (to be published).

[12] G. Yudin, S. Chelkowski, J. Itatani, A. Bandrauk, and P. Corkum, Phys. Rev. A 72, 051401 (2005).

[13] Y. H. Jiang et al., Phys. Rev. Lett. 105, 263002 (2010).

[14] Y.H. Jiang et al., Phys. Rev. A 81, 051402 (2010).

[15] Y.H. Jiang et al., Phys. Rev. A 81, 021401 (2010).

[16] C. Bostedt, H. N. Chapman, J. T. Costello, J. R. Crespo López-Urrutia, S. Düsterer, S. W. Epp, J. Feldhaus, A. 
Föhlisch, M. Meyer, and T. Möller, Nucl. Instrum. Methods Phys. Res., Sect. A 601, 108 (2009).

[17] http://www.elettra.trieste.it/FERMI.

[18] L. A. A. Nikolopoulos and P. Lambropoulos, J. Phys. B 39, 883 (2006)

[19] B. Sheehy, B. Walker, and L. F. DiMauro, Phys. Rev. Lett. 74, 4799 (1995).

[20] E. Charron, A. Giusti-Suzor, and F. H. Mies, Phys. Rev. Lett. 75, 2815 (1995).

[21] I. Franco and P. Brumer, J. Phys. B 41, 074003 (2008).

[22] F. Martín et al., Science 315, 629 (2007).

[23] J. L. Sanz-Vicario, H. Bachau, and F. Martín, Phys. Rev. A 73, 033410 (2006)
[24] J. L. Sanz-Vicario, A. Palacios, H. Bachau, and F. Martín, J. Electron Spectrosc. Relat. Phenom. 161, 182 (2007).

[25] D. Dowek, J. F. Pérez-Torres, Y. J. Picard, P. Billaud, C. Elkharrat, J. C. Houver, J. L. Sanz-Vicario, and F. Martín, Phys. Rev. Lett. 104, 233003 (2010).

[26] A. Zavriyev, P. Bucksbaum, J. Squier, and F. Saline, Phys. Rev. Lett. 70, 1077 (1993).

[27] S. Lee, J. Chem. Phys. 82, 4588 (1985).

[28] J. Ullrich, R. Moshammer, A. Dorn, R. Dörner, L.P. H. Schmidt, and H. Schmidt-Böcking, Rep. Prog. Phys. 66, 1463 (2003).

[29] A. González-Castrillo, J. F. Pérrez-Torres, A. Palacios, and F. Martín, Theor. Chem. Acc. 128, 735 (2010). 